\title{
Transmission Electron Microscopy and Theoretical Analysis of AuCu Nanoparticles: Atomic Distribution and Dynamic Behavior
}

\author{
J.A. ASCENCIO ${ }^{1 *}$ H.B. LIU, ${ }^{1}$ U. PAL,${ }^{2}$ A. MEDINA, ${ }^{3}$ AND Z.L. WANG ${ }^{4}$ \\ ${ }^{1}$ Programa de Investigación y Desarrollo de Ductos, Instituto Mexicano del Petroleo, \\ Eje Central Lázaro Cárdenas No. 152, Col. San Bartolo Atepehuacan, C.P.07730, Mexico D.F., Mexico \\ ${ }^{2}$ Instituto de Física, Universidad Autónoma de Puebla, Apdo. Postal J-48, Puebla, Pue. 72570, Mexico \\ ${ }^{3}$ Instituto de Investigaciones Metalúrgicas, UMSNH. Edificio U. Ciudad Universitaria, Morelia, Michoacán. 58000, Mexico \\ ${ }^{4}$ School of Materials Science and Engineering, Georgia Institute of Technology, Atlanta, Georgia $30332-0245$
}

\section{KEY WORDS bimetallic nanoparticles; high resolution electron microscopy; quantum mechan-} ics; molecular simulation

\begin{abstract}
Though the application of bimetallic nanoparticles is becoming increasingly important, the local atomistic structure of such alloyed particles, which is critical for tailoring their properties, is not yet very clearly understood. In this work, we present detailed study on the atomistic structure of $\mathrm{Au}-\mathrm{Cu}$ nanoparticles so as to determine their most stable configurations and the conditions for obtaining clusters of different structural variants. The dynamic behavior of these nanoparticles upon local heating is investigated. AuCu nanoparticles are characterized by high resolution transmission electron microscopy (HRTEM) and energy filtering elemental composition mapping (EFECM), which allowed us to study the internal structure and the elemental distribution in the particles. Quantum mechanical approaches and classic molecular dynamics methods are applied to model the structure and to determine the lowest energy configurations, the corresponding electronic structures, and understand structural transition of clusters upon heating, supported by experimental evidences. Our theoretical results demonstrate only the core/shell bimetallic structure have negative heat of formation, both for decahedra and octahedral, and energetically favoring core/shell structure is with Au covering the core of $\mathrm{Cu}$, whose reverse core/shell structure is not stable and may transform back at a certain temperature. Experimental evidences corroborate these structures and their structural changes upon heating, demonstrating the possibility to manipulate the structure of such bimetallic nanoparticles using extra stimulating energy, which is in accordance with the calculated coherence energy proportions between the different configurations. Microsc. Res. Tech. 69:522-530, 2006. @ 2006 Wiley-Liss, Inc.
\end{abstract}

\section{INTRODUCTION}

In the emerging field of new materials, designing, synthesis, and atomistic characterization of nanostructures have become very important, because the manipulation of structure has a direct effect in their macroscopic properties. In fact, the physical and chemical properties of nanoparticles are directly related to their chemical composition, size and volume, and surface structural characteristics. Therefore, an integrated investigation by combining experimental and theoretical approaches is vitally important for understanding their unique structures and properties. In the recent years, the synthesis of bimetallic nanoparticles has been very successful in obtaining small particles of multiple elements (De Meijer et al. 1997; Link et al. 1999; Ruiz et al. 2002), followed by analysis of their properties well supported by analytical (José-Yacamán et al. 2002) and theoretical methods (José-Yacamán et al. 2001; Liu et al. 2001). Particularly, the case of $\mathrm{AuCu}$ nanoparticles has attracted vast attention because of the possibility of using them in catalysis and other fields. Copper and gold alloyed structure is a typical example for investigating short-range ordering. In a previous work, we reported the synthesis and internal structure characterization of $\mathrm{AuCu}$ nanoparticles (Pal et al. 2004), identifying the main formation shapes as decahedrons and truncated octahedrons. The evidences demonstrated that the structure is directly related with the proportion of $\mathrm{Au}$ and $\mathrm{Cu}$ atoms. However, an important question that arises when the synthesis involves two different elements is how the elements are distributed in the particle and on the surface? The different configurations would imply significant differences in the possibility to apply them, since the exposed atoms and the corresponding electronic distribution will be varied in a large degree for different configurations. Gonella et al. (1999) have reported an interesting study about the stability of $\mathrm{AuCu}$ nanoparticles by ion implantation, and even

*Correspondence to: J.A. Ascencio, Programa de Investigación y Desarrollo de Ductos, Instituto Mexicano del Petroleo, Eje Central Lázaro Cárdenas No. 152, Col. San Bartolo Atepehuacan, C.P. 07730, Mexico D.F., Mexico.

E-mail: ascencio@ imp.mx

Received 13 June 2004; accepted in revised form 1 December 2004 DOI 10.1002/jemt.20321

Published online 26 May 2006 in Wiley InterScience (www.interscience.wiley.com). 
when they claim that the production of core-shell systems are consequence of the previous alloy formed clusters, they propose the highly stable structures only when a shell of Au over a core of $\mathrm{Cu}$; however, this study is based on the annealing in oxygen and hydrogen atmospheres, where the effects of interaction with the gases contribute to the final structure.

Because of the size and structural complications of nanoparticles, the understanding of this problem has not been addressed satisfactorily. Fortunately, the modern advanced analysis techniques using electron microscopy (José-Yacamán et al. 2002), together with the actual capacity of computation that allows the use of quantum mechanics and molecular dynamics for the study of relatively big clusters (Liu et al. 2001), provide a powerful approach for investigating the structure of nanoparticles. It is now possible to match experimental results with theoretical calculations for a better understanding of the structure.

In this work, we study the atomistic structure of $\mathrm{AuCu}$ nanoparticles with help of theoretical modeling and experimental methods, particularly high resolution electron microscopy (HRTEM) and energy filtering elemental composition mapping. The stable configurations and their behavior upon energy stimulation process are studied for $\mathrm{AuCu}$ clusters of different compositions.

\section{METHODS Experimental}

The synthesis of bimetallic $\mathrm{Au}-\mathrm{Cu}$ colloidal particles was made by simultaneous reduction of corresponding metal chloride salts in presence of polyvinyle-pyrrolidone (PVP), as reported in detail elsewhere (Pal et al. 2004). Structural characterization of the samples was made using a Schottky field emission Jeol JEM 2010-F microscope with analytical equipments attached. The samples were prepared by spreading a droplet of colloidal solution of $\mathrm{AuCu}$ nanoparticles onto a carbon film supported by a $\mathrm{Cu}$ grid and subsequent drying in vacuum. The samples were analyzed by high resolution microscopy and the elemental composition was mapped by high angle annular dark field imaging and the energy filtering technique. The high resolution electron microscopy (HREM) images were processed by digital methods in the real and Fourier spaces.

\section{Theoretical}

To determine the electronic structure and orbital distribution differences among core-shell clusters and $\mathrm{AuCu}$ alloy-based nanoparticles, we used the DMol3 method, which is a quantum mechanical method based on the density functional theory (DFT); we have also used a local density approximation with the Perdew Wang exchange-correlation functional (Delley, 1990, 2000; DMol3, 1999; Perdew et al. 1996). Geometry optimization and a single point energy calculation were performed in order to identify the lowest energy configuration with its highest occupied and lowest unoccupied molecular orbitals (HOMO and LUMO respectively) distributions for both the pure and bimetallic core-shell and alloyed cluster configurations. The evaluation of the HOMO and LUMO gap allows understanding the optical, electronic, and chemical selectiv- ity characteristics of small clusters, which help us in the understanding of the physicochemical properties of the materials.

For bigger clusters, molecular dynamics simulation was employed. Interatomic interactions between $\mathrm{Au}$ and $\mathrm{Cu}$ were described by a simple analytical embedded-atom method (EAM) developed by Cai and Ye (1996). The model includes a long-range force. In this model, electron-density function is taken as decreasing exponential function; two-body potential is defined as a function given by Rose et al. (1984), and embedding energy is assumed to be a universal form suggested by Banerjee and Smith (1988). The alloy model of Johnson (1989) is applied and an extra parameter is introduced in order to fit dilute-limit heats of solution. For the $\mathrm{AuCu}$ compounds, the predicted heats of formation are in reasonable agreement with first principle calculations and experimental data, and consistent lattice constants are predicted. The $\mathrm{Au}-\mathrm{Cu}$ alloying potential can be used in a wide range of components with a great accuracy. The MD simulations were performed using XMD developed by Riffkin (2003). The program employs a predictor-corrector algorithm to integrate the equation of motion. A time step of $5 \times 10^{-15} \mathrm{~s}(5 \mathrm{fs})$ was used.

\section{RESULTS AND DISCUSSION Nanoparticle Characterization}

TEM images were obtained for the samples in order to study the size distribution of the produced particles, which usually depends on the reduction method and the elemental composition. Three different proportions of $\mathrm{Au}$ and $\mathrm{Cu}$ were used and evaluated by these analyses, demonstrated in an earlier report (Pal et al. 2004) that both compositions produce small particles. In Figure 1, a low magnification TEM image for the $\mathrm{Au}-\mathrm{Cu}$ samples is shown together with a size distribution plot for the $\mathrm{Au}-\mathrm{Cu}$ proportions cluster synthesized. It is clear that the smallest particles are obtained for $\mathrm{AuCu}$, but in all the cases we found aggregates smaller than $15 \mathrm{~nm}$.

\section{Stability of Nanoparticles}

It is well known that in practice the different configurations of nanoparticles coexist in a sample because not in all of the cases the clusters would be in the minimized energy configurations. However, an important parameter we are including in this study is the atomistic distribution of the elements, inducing a significant difference on the electronic structure, which has a direct influence over the chemical and physical properties, as can be observed by a simple inspection of frontier orbital distributions (and values) for $\mathrm{Au}$-core/Cushell $(\mathrm{Au} / \mathrm{Cu})$ and $\mathrm{Cu}$ core/Au-shell $(\mathrm{Cu} / \mathrm{Au})$ and $\mathrm{AuCu}$ alloy-like structures. In Figure 2 we show the results of a DFT calculation for the minimized energy configurations of the eight different clusters. The figure includes the geometry optimized model and the corresponding electrostatic potential distribution for the structures: (a) $\mathrm{Au}_{55}$, (b) $\mathrm{Cu}_{55}$, (c) $\mathrm{Au}_{13} \mathrm{Cu}_{42}$, (d) $\mathrm{Cu}_{13} \mathrm{Au}_{42}$ coreshells and four more structures of the type of alloys, considering the (e) $\mathrm{Au}_{36} \mathrm{Cu}_{19}$, (f) $\mathrm{Au}_{19} \mathrm{Cu}_{36}$ configurations that are based $\mathrm{AuCu}_{3}$ alloy and correspond to a concentric variation of elements, while the (g) 

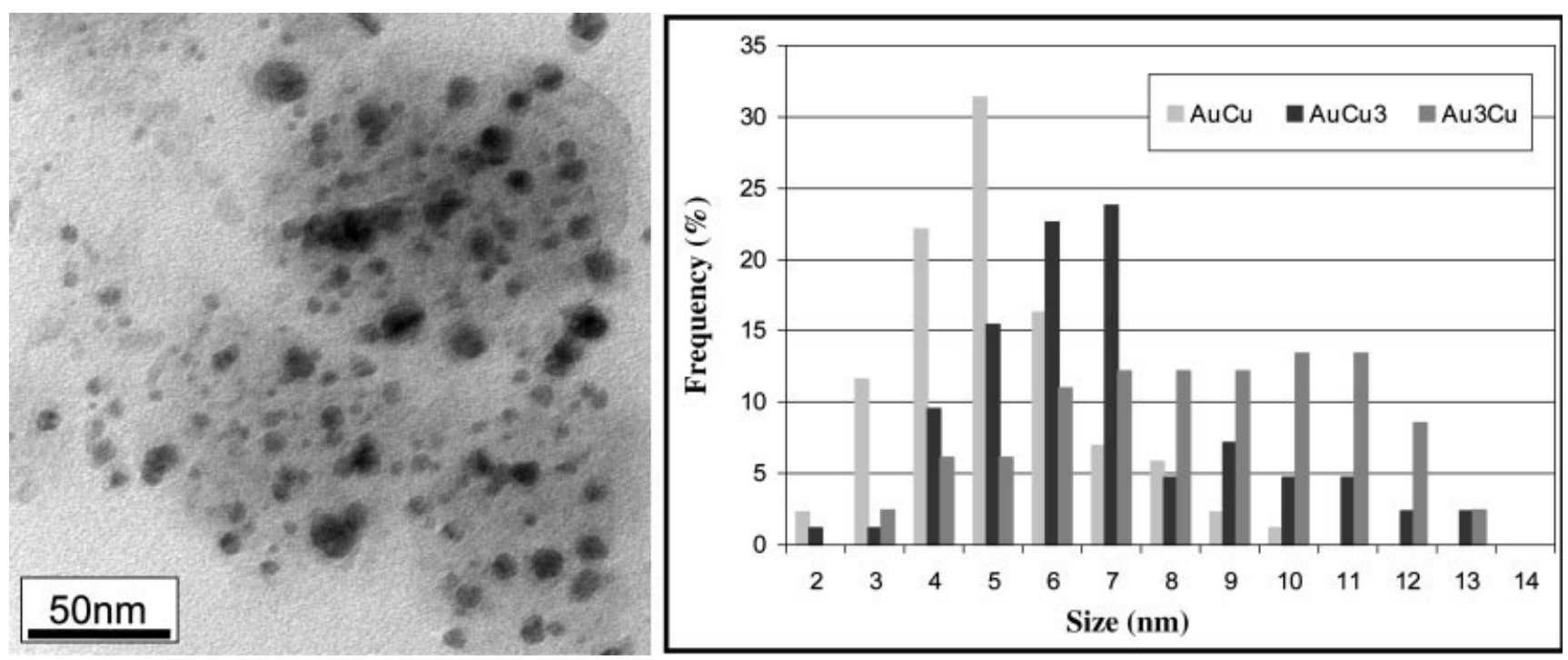

Fig. 1. TEM image of $\mathrm{Au}-\mathrm{Cu}$ nanoparticles besides the size distribution plot for the three different synthesized samples.

$\mathrm{Au}_{24} \mathrm{Cu}_{31}$, and $(\mathrm{h}) \mathrm{Au}_{31} \mathrm{Cu}_{24}$ that are planes based on the $\mathrm{AuCu}$ alloys. For clarity, the structures are shown in the [001] crystallographic view.

From the models we can identify that the pure element structures show minimum shape distortion, while the presence of two different elements induce important increasing of distortion mainly inducing a reduction of distance around the $\mathrm{Cu}$ atoms concentrically with exception of the two last structures that show the distortion per planes. Besides, the electrostatic potential distribution iso-surfaces denote a big homogeneity with well identified local negative sites in the central atoms of the $\{001\}$ faces, which are bigger for the alloyed nanoparticles, and it is especially interesting the polarization produced in the layered alloys that show no concentric symmetry.

The values for the HOMO, LUMO, and the corresponding gaps are shown in Table 1 . Similarly, in the table the binding and the corresponding coherence energy are shown. The binding energy shows a contribution without element distinction and the energy required to form the bimetallic system allows understating better the stability of this kind of aggregates. In this way the most stable structures is the $\mathrm{Au}_{19} \mathrm{Cu}_{36}$ alloyed cluster, while there are also two very stable configurations; the core-shell of $\mathrm{Au}_{42} \mathrm{Cu}_{13}$ that correspond to $\mathrm{Au}$ external atoms and the layered alloy with $\mathrm{Au}_{31} \mathrm{Cu}_{24}$. It must be considered that these properties depend on the number of atoms, the corresponding size, and the atomistic distribution. These parameters are crucial in the formation of each type of nanoparticles (José-Yacamán et al. 2001). Besides, it is clear that the highest gap is for the case of $\mathrm{Au}_{13} \mathrm{Cu}_{42}$ core shell structure that implies the high probability to be produced during a chemical synthesis (Vinod et al. 1998). The big difference between identified gaps represents an important electronic behavior, because for the different configurations, it predicts a quite different hardness for the material, which involves completely different kinds of induced catalysis effect (Gonzalo,
2003; Shaikhutdinov et al. 2003) and behavior in electronic devices (Ruiz et al. 2002).

The structural configuration of nanoparticles (clusters) is determined by several factors such as the number of atoms and the energy variation between the constituent atoms. The apparently less probable structural configuration will have a higher energy. We believe that during the process of a chemical synthesis of nanoparticles, it is possible to produce both stable and less stable clusters. However, the production of the most stable configuration depends on the control of the atomic proportion, which is determined by the original precursor stoichometry. This indicates that the configuration of the clusters may be subjected to a dynamic change until a most stable configuration is reached.

To determine the morphology and tendency of the formation of $\mathrm{Au}-\mathrm{Cu}$ bimetallic clusters, 262-atom decahedra and 861-atom octahedra with different composition and distributions were built for the afore-mentioned classic molecular dynamics simulation. These structures (Tables 2 and 3) include eutectic-like and core-shell-like configurations. In Tables 2 and 3, the total cohesive energy, stoichiometric energy, and the corresponding formation energies are shown in order to determine the possibility to produce each one of them for both the cases (truncated octahedron and decahedron respectively). Our results revealed that only the core-shell like bimetallic structures have negative heat of formation, both for decahedra and octahedral, and the energetically favoring core-shell structure is with Au covering the core of $\mathrm{Cu}$. However, the differences between the energies for all of the configurations are relatively low and the coexistence of these structures in synthesized samples must be common. As the critical number of atoms needed to produce each type of nanoparticles, the formation of both decahedrons and octahedrons is probable in the material, and the synthesis conditions will determine the kind of configuration based on the energy surface reduction capacity. 

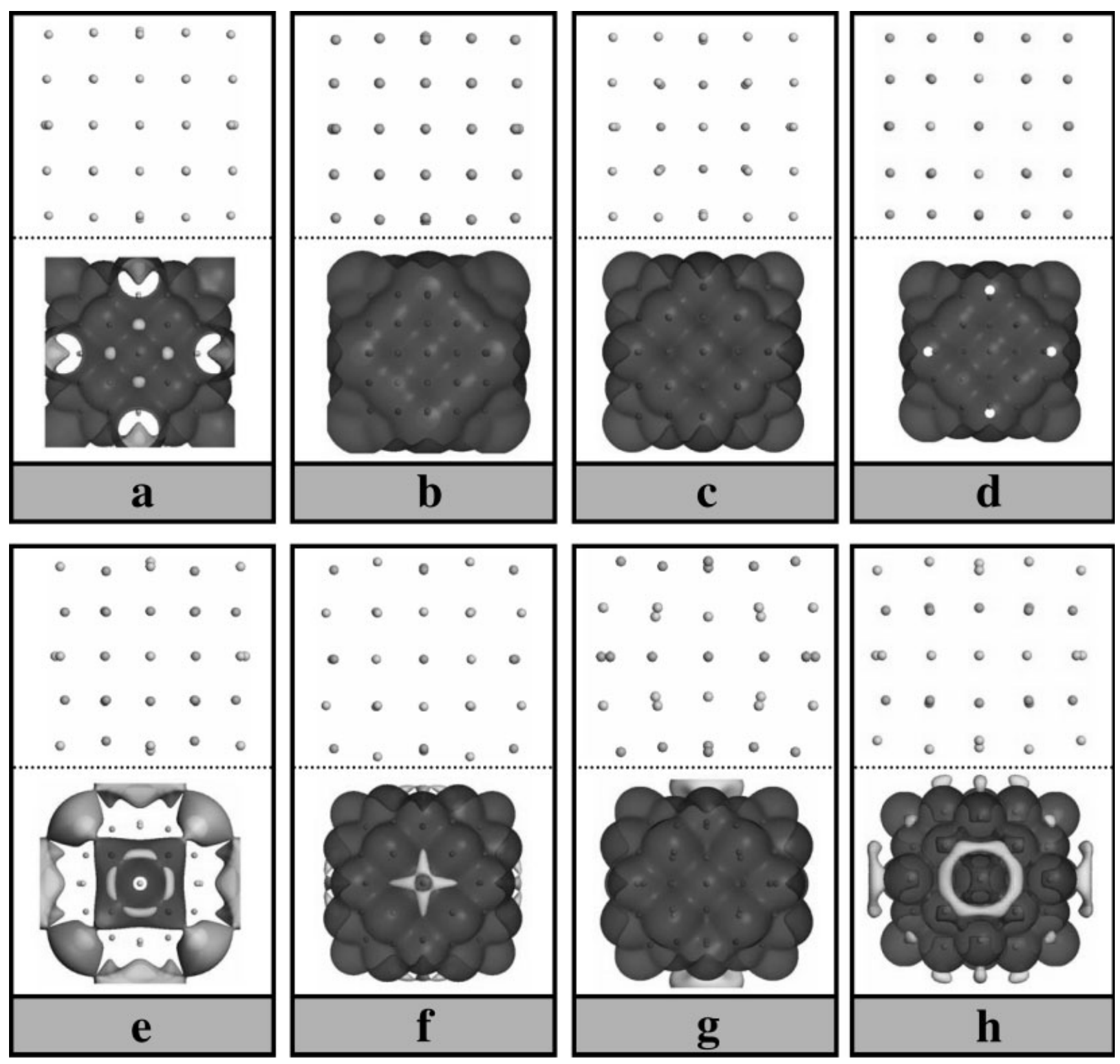

Fig. 2. Quantum mechanics predicted structure models by energy minimization, HOMO and LUMO distributions (a) $\mathrm{Au}_{55}$, (b) $\mathrm{Cu}_{55}$, (c) $\mathrm{Au}_{13} \mathrm{Cu}_{42}$ (Au-core/Cu-shell), (d) $\mathrm{Cu}_{13} \mathrm{Au}_{42}$ (Cu-core/Au-shell), (e) $\mathrm{Au}_{36} \mathrm{Cu}_{19}$, (f) $\mathrm{Au}_{19} \mathrm{Cu}_{36}$ that correspond to concentric alloy, while (g) $\mathrm{Au}_{24} \mathrm{Cu}_{31}$ and (h) $\mathrm{Au}_{31} \mathrm{Cu}_{24}$ are based on a layered $\mathrm{AuCu}$ alloy.

For embedding atom model, the alloying pair potential determines the magnitude of chemical order. From our previous report it was seen that $\mathrm{Au}-\mathrm{Cu}$ alloying pair potential is very close to the mathematics average of $\mathrm{Au}-\mathrm{Au}$ and $\mathrm{Cu}-\mathrm{Cu}$ pair potential, which indicates that the bimetal formation potential is very small and it must not affect the bimetal formation energy significantly. The other factor affecting bimetal formation is the incoherence of the structure, which may dominate the process of bimetallic aggregation and configuration. In fact, the nearest neighbor distances in $\mathrm{AuCu}$ alloy system are different from that of pure $\mathrm{Au}$ and $\mathrm{Cu}$ by $11.4 \%$ and $12.8 \%$, respectively, which are large enough to generate a high structure incoherent energy. There exists structural incoherency for all of the structures treated here and the core-shell structures, where $\mathrm{Au}$ covering $\mathrm{Cu}$ has the minimum. This effect is attributed to the contraction of Au surface, leading to a good accommodation to the $\mathrm{Cu}$ core, which has a smaller nearest neighboring distance, hence producing a small structural mismatch for $\mathrm{Cu}$ Core. For the core-shell with $\mathrm{Cu}$ covering $\mathrm{Au}, \mathrm{Au}$ core has a maximum structure incoherent energy. Generally speaking, structural incoherency dominates the formation of $\mathrm{Au}-\mathrm{Cu}$ bimetal 
TABLE 1. HOMO, LUMO, and Gap values for the three configurations calculated by quantum mechanics

\begin{tabular}{lccrr}
\hline Model & Big energy & HOMO (eV) & LUMO (eV) & $\begin{array}{r}\text { Formation energy } \\
\text { of bimetallic }(\mathrm{eV})\end{array}$ \\
\hline Au55 & -139.965 & -4.3823 & -4.0283 & 0 \\
Cu55 & -186.861 & -6.3042 & -5.9851 & 0.3539 \\
AuI3Cu42 & -159.715 & -5.7613 & -5.3541 & 0.3191 \\
Au42CuI3 & -148.102 & -6.0232 & -5.9120 & 0.4072 \\
Aul9Cu36 & -169.219 & 4.4606 & -4.0669 & 0.1113 \\
Au36Cul9 & -149.243 & -5.1299 & 4.9616 & 0.3937 \\
Au24Cu31 & -158.907 & 4.3462 & 4.1203 & 0.1683 \\
Au31Cu24 & -156.524 & -3.0233 & -2.6777 & 0.2259 \\
\hline
\end{tabular}

TABLE 2. The formation energy of Au-Cu bimetallic clusters: Decahedron with 262 atoms

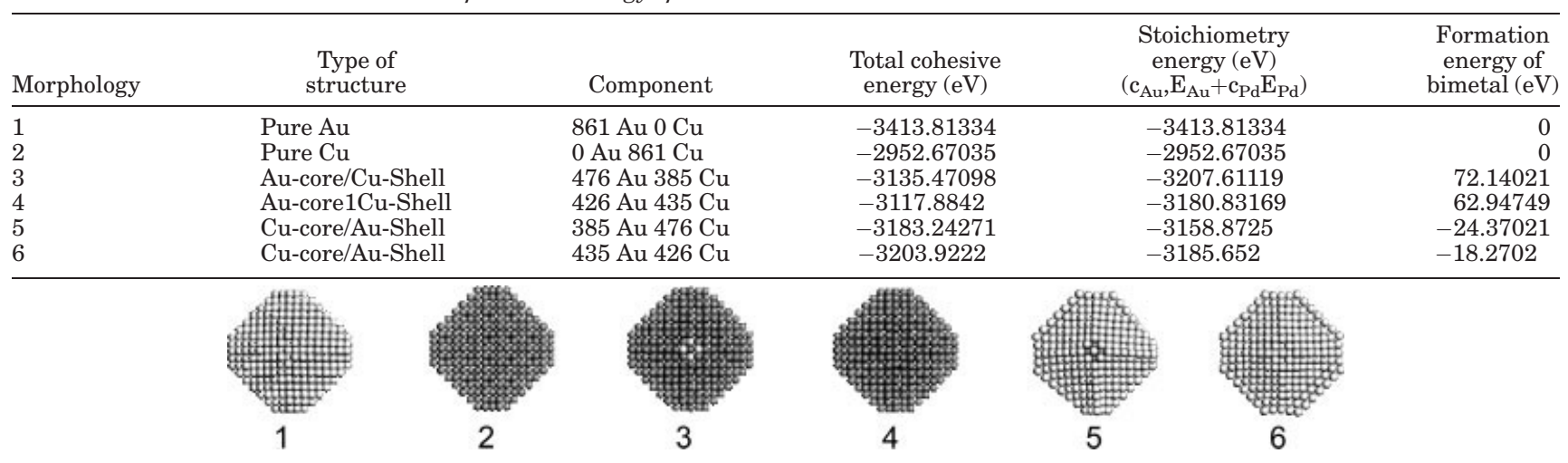

TABLE 3. The formation energy of Au-Cu bimetallic clusters: Octahedron with 861 atoms

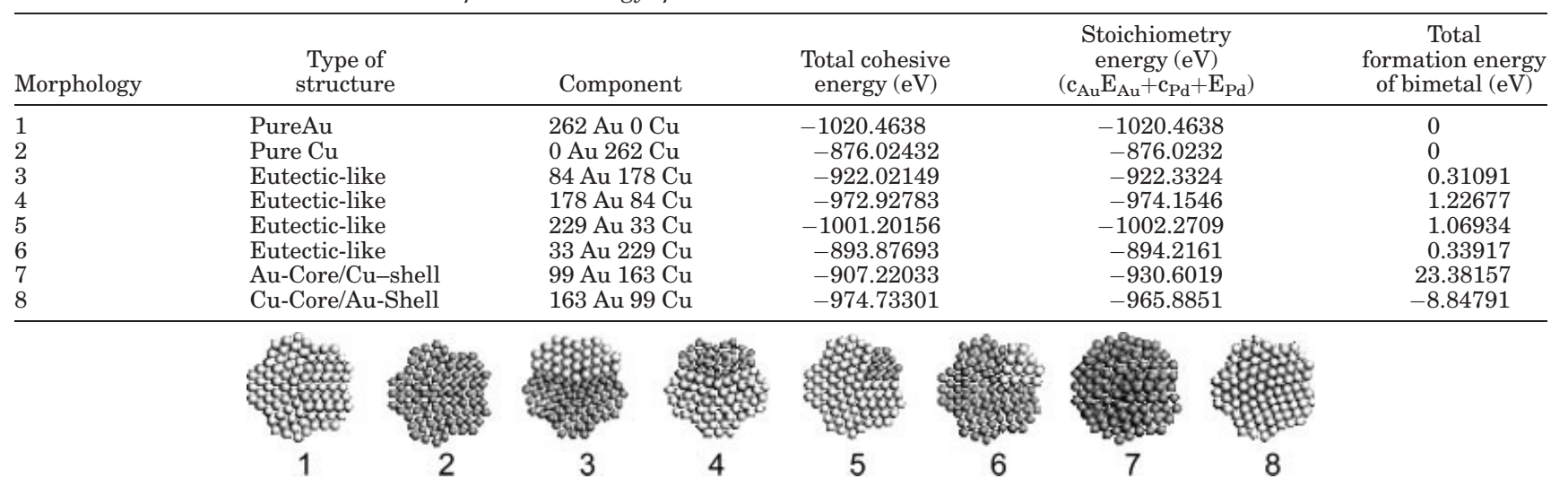

cluster, and core-shell with Au covering $\mathrm{Cu}$ is energetically favorable.

To confirm the mentioned theoretical results, $\mathrm{AuCu}$ nanoparticles were analyzed by HREM, searching for different structures. In Figure 3, a couple of multiple twined nanoparticles are shown besides two truncated octahedron clusters. The shape and contrast are quite similar to the single element nanoparticles and the distances show a homogeneous distribution of values between both the $\mathrm{Au}$ and $\mathrm{Cu}$, while just small differences are observed near to the edge of the particles. This behavior was expected as the difference in lattice constants between the two metals is small.

However, obtaining direct evidences of the elemental composition variations are not easy. We used the high angle annular dark field imaging in scanning TEM to identify the distribution of Au atoms, which are no easy to be distinguished from the $\mathrm{Cu}$ because of the small $\mathrm{Z}$ number difference as well as the variation in projected sample thickness, as can be seen in Figure 4a. It is likely that both elements are in the observed region. Energy filtering mapping method was used in order to recognize the core shell distribution, after a false color contrast. An elemental mapping for a big area is shown in Figure 4c, where different colors correspond to different elements; this must be considered that the contrast was improved for separating the signals of $\mathrm{Au}$ and $\mathrm{Cu}$. It is clear from the image that there are regions with dispersed $\mathrm{Cu}$, and the $\mathrm{Au}$ forms well defined clusters. The $\mathrm{Au}$ core- $\mathrm{Cu}$ shell configuration is 

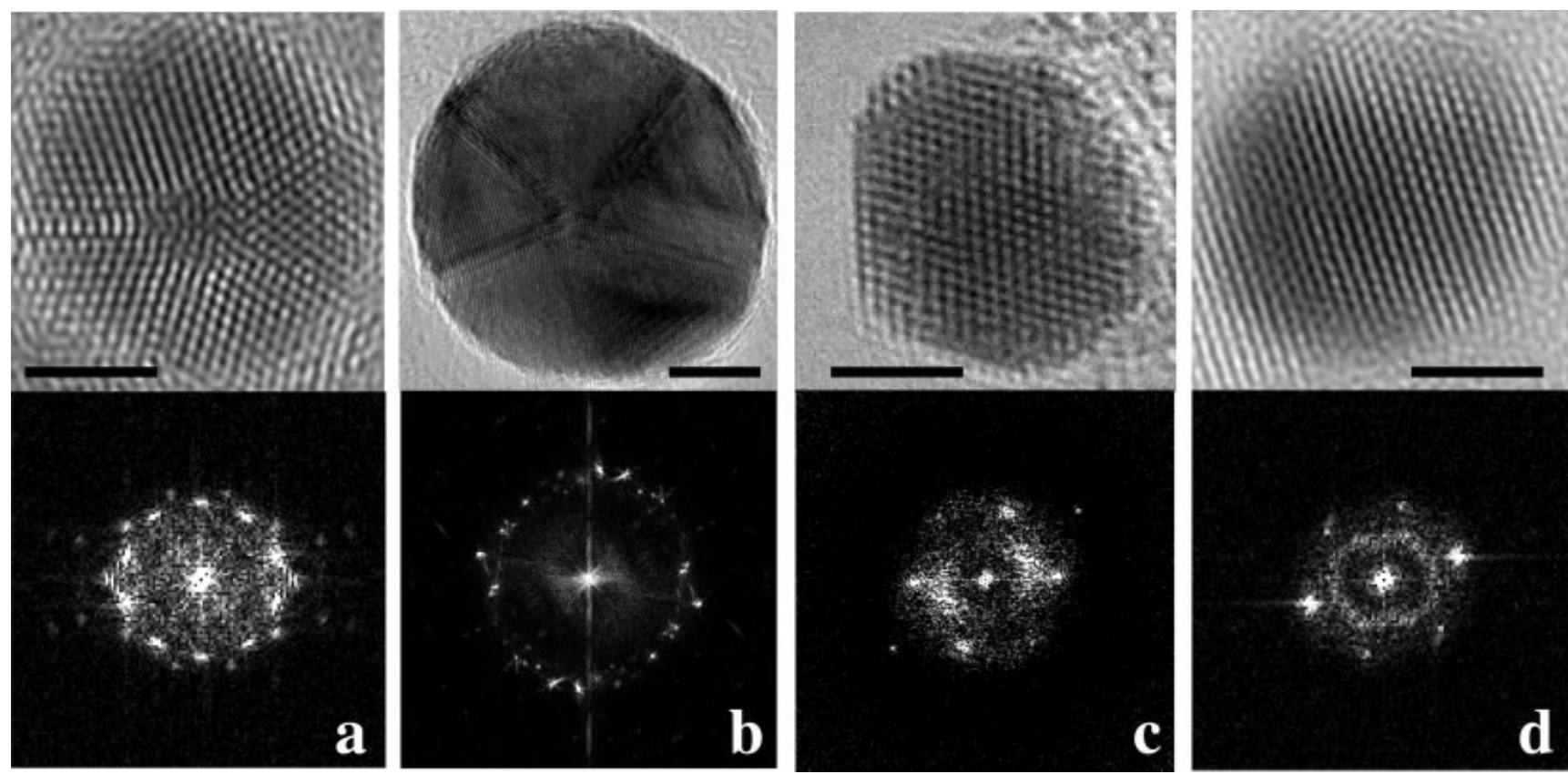

Fig. 3. HRTEM images from a couple of decahedron (a, b) and two fcc-like structures (c, d) with their corresponding FFT pattern. Scale bar corresponds to $2 \mathrm{~nm}$ in all the images.

clearly observed in the image for several particles. A couple of modeled structures are included inside the figure for clarity. This analysis allows us to confirm by a simple way the existence of bigger nanoparticles of $\mathrm{Au}$ core and $\mathrm{Cu}$ shell (marked with arrows) and the presence of common single element and alloy configurations in small clusters.

The size and internal energy must be the determining factors for the coexistence of these clusters. However, for the smallest particles, the influence of the electron beam energy might be enough to produce perturbations in their shape and structure, which has been demonstrated in previous works (Ascencio et al. 2001, 2002; Liu et al. 2002, 2003). Effect of the electron beam over the clusters is equivalent to an increase in temperature up to $100-200^{\circ} \mathrm{C}$, interesting factor for the smallest clusters where the electron beam produces quasi-melting effects and even coalescence (Liu et al. 2003).

To understand the atomistic behavior of $\mathrm{Au} / \mathrm{Cu}$ nanoparticles under dynamical processes, calculations are made for the 262 atoms decahedron and the 861 atoms octahedron, core-shell configurations. In the Figure 5, the plots of the corresponding calculated cohesive energy versus temperature are shown for the cases of $\mathrm{Au} / \mathrm{Cu}$ and also for $\mathrm{Cu} / \mathrm{Au}$ configurations. The analysis shows a quite interesting effect for the clusters of $\mathrm{Au}$ core- $\mathrm{Cu}$ shell structures that present a singularity when the temperature passes through $550 \mathrm{~K}$ to $600 \mathrm{~K}$, where the energy suddenly decreases from $-3.29 \mathrm{eV}$ to $-3.40 \mathrm{eV}$ for the $\mathrm{Au}_{99} \mathrm{Cu}_{163}$ decahedron (Fig. 5a). A similar effect is observed for the octahedron when the $\mathrm{Au}_{385} \mathrm{Cu}_{476}$ reaches $620 \mathrm{~K}$ until $680 \mathrm{~K}$, reducing its energy suddenly from $-3.48 \mathrm{eV}$ to $-3.55 \mathrm{eV}$. These changes are not observed for the $\mathrm{Cu}$ core-Au shell case, where the energy increases with an almost constant rate as it was expected for both the structures.

Through the observation on the trajectories of the earlier mentioned processes, it is found that the sudden drops in energy for $\mathrm{Au}$ core- $\mathrm{Cu}$ Shell structure are induced by the position reversions of shell atom and core atom, that is, the atoms $\mathrm{Au}$ on shell enter into the core and finally are covered by atoms $\mathrm{Cu}$. It demonstrates from the point of view of dynamics our conclusion that $\mathrm{Au}$ core/Cu shell structures are not stable and can not exist at a certain high temperature.

To acquire experimental evidence of the afore mentioned structural transition, we used the same electron beam in the microscope, focusing the spot on a small nanoparticle. The Figure 6 shows the sequence of a cluster with originally fcc like contrast, and two posterior stages after 2 and 5 min of electron beam irradiation. We improved the contrast of the particle and enhanced the internal structure by means of a digital processing in the real and Fourier spaces. It is clear that the parallel lines of the early stage cluster (Fig. 6a) correspond to that of the fcc particle, while in the second stage the defects appear (Fig. 6b); however, the corresponding image of the cluster after 5 min of irradiation shows significant structural change, which is more associated to multiple twined nanoparticles.

These evidences demonstrate the structural transformation in $\mathrm{AuCu}$ nanoparticles, and suggest that the HOMO-LUMO gap, chemical potential, and the reaction capacity of the bimetallic nanoparticles can be locally modified by a simple local heating process.

This observed effect is entirely related to the structure energy incoherency calculated for the clusters, which must dominate the total energy of the clusters when there is extra stimulating energy, reducing the 

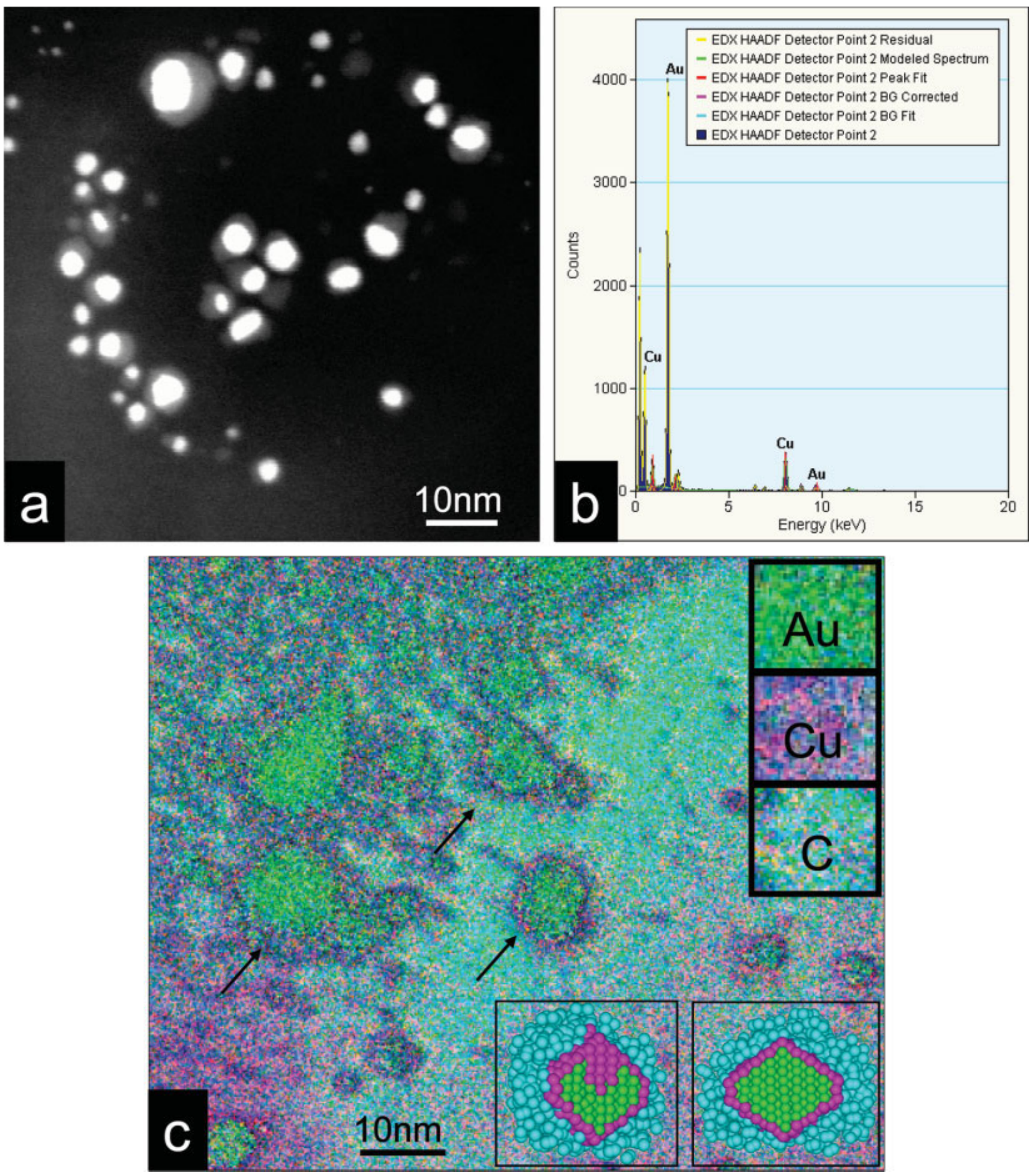

Fig. 4. TEM image for analyzing elemental mapping in the nanoparticles by using an energy filter. A couple of models are included in the right inset.

internal strain and finding the lowest energy configuration. This behavior has been widely studied for single element nanoparticles; however, the observation of this effect in bimetallic nanoparticles is new and is influenced by the different atomic radius of components. Such structural changes of bimetallic nanoparticles have significant impact to their applications. 

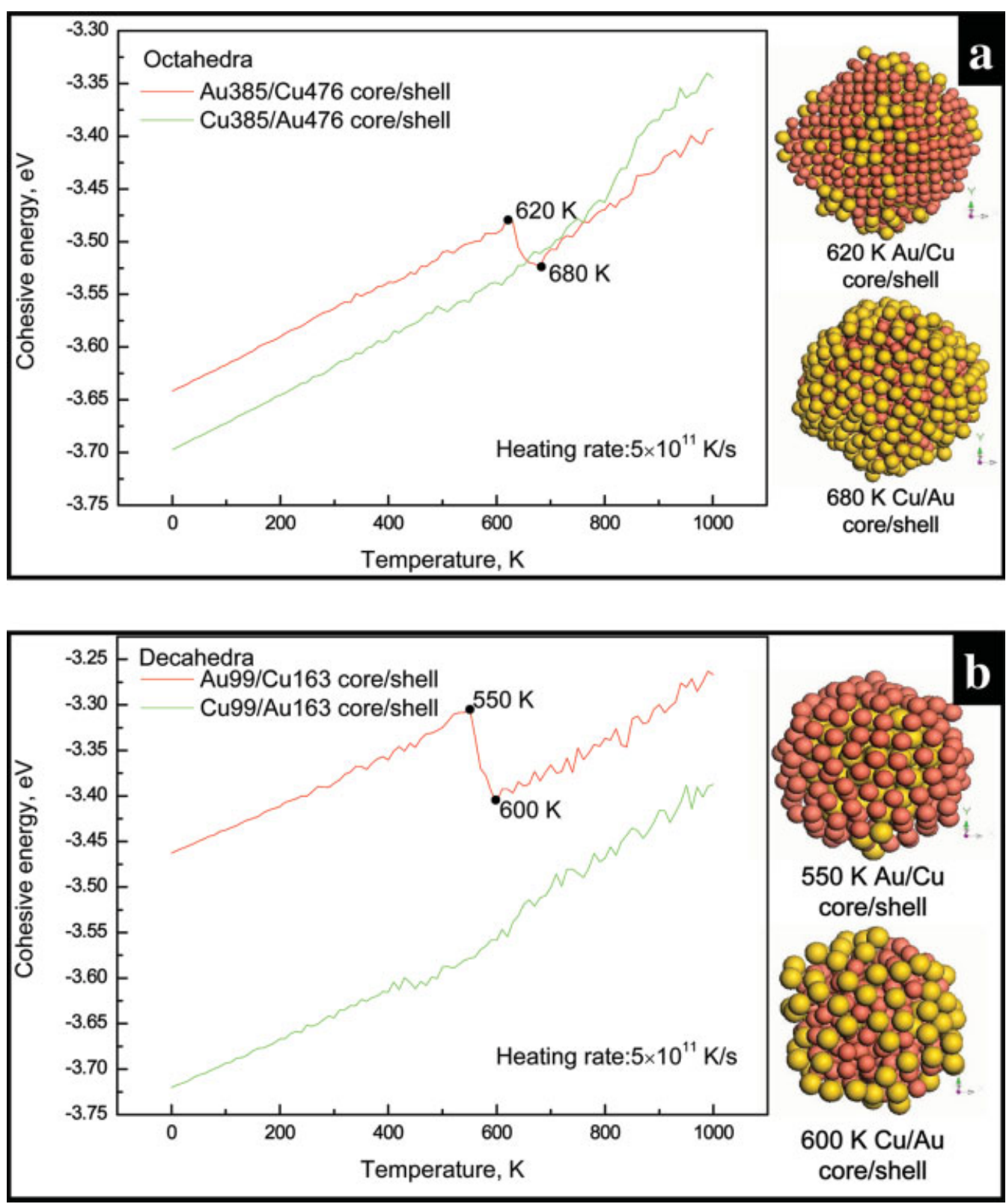

Fig. 5. Cohesive energy per atom versus temperature (a) for decahedra with core/shell structures of Au99/Cu163 and Cu99/Au163; (b) for octahedra with core/shell structures of Au385/Cu476 and Cu385/ Au476.

\section{CONCLUSIONS}

Through quantum mechanical calculations, we have demonstrated the differences between nanoparticles with same number constituting atoms but different atomic distributions. While the energy invested to form bimetallic clusters is lower for a couple of alloy-like structures and the $\mathrm{Au}$ shell over the $\mathrm{Cu}$ core, the HOMO-LUMO gap suggest the possibility to generate an $\mathrm{Au}$ core $\mathrm{Cu}$ shell configuration. Our analysis reveals that the structural incoherency dominates the formation of $\mathrm{Au}-\mathrm{Cu}$ bimetallic clusters, and the core-shell is the energetically most favorable configuration. Even the $\mathrm{Cu}$ core-Au shell structure is more favorable, other structures may coexist in the synthesized bimetallic colloids because of the small difference between the configuration energies of different structures. The theoretical prediction of coexistence of fcc-like and multiple twined $\mathrm{AuCu}$ nanoparticles similar to the case of reported monometallic nanoparticles is supported by the experimental observations. We could identify the existence of octahedral and decahedral $\mathrm{AuCu}$ clusters experimentally. Even when the classical calculations and previous authors consider not possible, we demonstrate the existence of $\mathrm{Au}$ core- $\mathrm{Cu}$ shell structures along, by using energy filter images, with other configurations in synthesized colloidal $\mathrm{AuCu}$ clusters. 

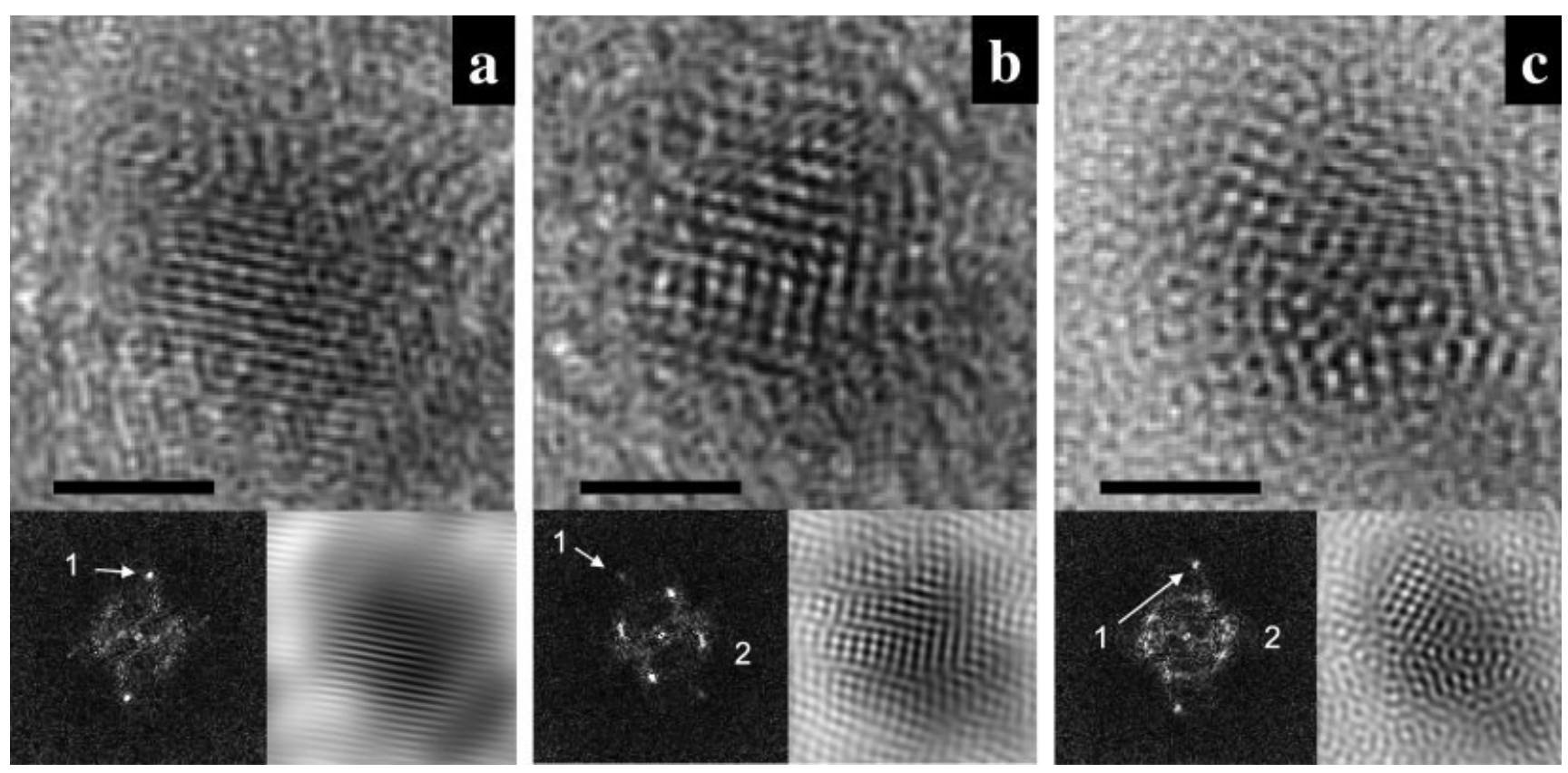

Fig. 6. Sequence of TEM images of a small $\mathrm{AuCu}$ particle, including its corresponding FFT and the frequency based filtered images from the marked dots. (a) Original particle with no evident defects, (b) Particle after 2 min electron irradiation with small defects, and (c) particle after 5 min showing multiple defects. Scale bar corresponds to $2 \mathrm{~nm}$ in all the images.

During theoretical heating processes, it was found that $\mathrm{Au}$ core $\mathrm{Cu}$ shell structures are not stable and can not persist, and they suddenly transform into its core shell reversion structure at a certain high temperature. On exposing the nanoparticles to the electron beam, we have observed a similar dynamic process.

With the help of theoretical and experimental methods, we report here an in-depth analysis of atomistic distribution in $\mathrm{Au}-\mathrm{Cu}$ nanoparticles, including their stability and dynamical behavior.

\section{ACKNOWLEDGMENTS}

We acknowledge the help of J.F. Sanchez Ramirez for his help in preparing $\mathrm{Au} / \mathrm{Cu}$ colloidal clusters and to $\mathrm{L}$. Rendon for technical support in HRTEM images.

\section{REFERENCES}

Ascencio JA, Pérez M, Tehuacanero S, José-Yacamán M. 2001. Experimental study of instabilities on metal nanoparticles a new kind of quasimelting. Appl Phys A: Mater Sci Process 73:295-301.

Ascencio JA, Mendoza M, Santamaría T, Pérez M, Nava I, GutiérrezWing C, José-Yacamán M. 2002. Structural instabilities in passivated gold nanoclusters induced by electron irradiation. J Cluster Sci 13:189-197.

Banerjea A, Smith JR. 1988. Origins of the universal binding-energy relation. Phys Rev B: Condens Matter Mater Phys 37:6632-6645.

Cai J, Ye YY. 1996. Simple analytical embedded-atom-potential model including a long-range force for fcc metals and their alloys. Phys Rev B: Condens Matter Mater Phys 54:8398-8410.

Delley B. 1990. An all-electron numerical method for solving the local density functional for polyatomic molecules. J Chem Phys 92:508516.

Delley B. 2000. From molecules to solids with the $\mathrm{DMol}^{3}$ approach. J Chem Phys 113:7756-7761.

De Meijer RJ, Stapel C, Jones DG, Roberts PD, Rozendaal A, Macdonald WG, Chen KZ, Zhang ZK, Cui ZL, Zuo DH, Yang DZ. 1997. Catalytic properties of nanostructured hydrogen storage nickel particles with cerium shell structure. Nanostruct Mater 8:205-213.

$\mathrm{DMol}^{3}$ module of Cerius ${ }^{2}$. Accelrys, San Diego. 1999.
Gonella F, Mattei G, Mazzoldi P, Sada C, Battaglin , Cattaruzza E. 1999. Au-Cu alloy nanoclusters in silica formed by ion implantation and annealing in reducing or oxidizing atmosphere. Appl Phys Lett 75:55-57.

Gonzalo J, Serna R, Solís J, Afonso CN, Babonneau D. 2003. Morphological and interaction effects on the surface plasmon resonance of metal nanoparticles. J Phys: Condens Matter 15:S3001-S3010.

Johnson RA. 1989. Alloy models with the embedded-atom method. Phys Rev B: Condens Matter Mater Phys 39:12554-12558.

José-Yacamán M, Ascencio JA, Liu HB. 2001. The structure and shape and stability of nanometric sized particles. J Vac Sci Techn B 19:1091-1103.

José-Yacamán M, Ascencio JA, Tehuacanero S, Marin M. 2002. Modern application of electron microscopy to catalysis. Top Catal 18:167-173.

Link S, Burda C, Wang ZL, El-Sayed MA. 1999. Electron dynamics in gold and gold-silver alloy nanoparticles: The influence of a nonequilibrium electron distribution and the size dependence of the electron-phonon relaxation. J Chem Phys 11:1255-1262.

Liu HB, Ascencio JA, Pérez M, José-Yacamán M. 2001. Melting behavior of intermediate sized gold isomers. Surf Sci 491:88-98.

Liu HB, Perez R, Canizal G, Ascencio JA. 2002. Stability and phase transition of sodium nanoclusters. Surf Sci 518:14-20.

Liu HB, Jose-Yacamán M, Perez R, Ascencio JA. 2003. Studies of nanocluster coalescence at high temperature. Appl Phys A: Mater Sci Process 77:63-67.

Pal U, Sanchez Ramirez JF, Liu HB, Medina A, Ascencio JA. 2004. Synthesis and structure determination of bimetallic $\mathrm{Au} / \mathrm{Cu}$ nanoparticles. Appl Phys A: Mater Sci Process 79:79-84.

Perdew JP, Burke K, Ernzerhof M. 1996. Generalized gradient approximation made simple. Phys Rev Lett 77:3865-3868.

Riffkin J. 2003. http://ims.uconn.edu/centers/simul

Rose JH, Smith JR, Guinea F, Ferrante J. 1984. Universal features of the equation of state of metals. Phys Rev B: Condens Matter Mater Phys 29:2963-2967.

Ruiz A, Arbiol J, Cirera A, Cornet A, Morante JR. 2002. Surface activation by $\mathrm{Pt}$-nanoclusters on titania for gas sensing applications. Mater Sci Eng C 19:105-109.

Shaikhutdinov SK, Meyer R, Naschitzki M, Bäumer M, Freund H. 2003. Size and support effects for CO adsorption on gold model catalysts. Catal Lett 86:211-213.

Vinod CP, Kulkarni GU, Rao CN. 1998. Size-dependent changes in the electronic structure of metal clusters as investigated by scanning tunneling spectroscopy. Chem Phys Lett 289:329-330. 